Voix et Images

voixetimages

\title{
Bibliographie de Yves Beauchemin
}

\section{Frances J. Summers et Thérèse Pouliot}

Volume 12, numéro 3 (36), printemps 1987

Yves Beauchemin

URI : https://id.erudit.org/iderudit/200656ar

DOI : https://doi.org/10.7202/200656ar

Aller au sommaire du numéro

Éditeur(s)

Université du Québec à Montréal

ISSN

0318-9201 (imprimé)

1705-933X (numérique)

Découvrir la revue

Citer ce document

Summers, F. J. \& Pouliot, T. (1987). Bibliographie de Yves Beauchemin. Voix et Images, 12(3), 416-428. https://doi.org/10.7202/200656ar d'utilisation que vous pouvez consulter en ligne.

https://apropos.erudit.org/fr/usagers/politique-dutilisation/ 


\title{
Bibliographie de Yves Beauchemin
}

\author{
par Frances J. Summers, Canberra, Australie \\ (avec la collaboration de Thérèse Pouliot, Université Laval)
}

\section{OEUVRES D'YVES BEAUCHEMIN}

\section{Romans}

L'Enfirouapé, Montréal, la Presse, 1974, $250 \mathrm{p}$.

L'Enfirouapé, Montréal, Stanké, Collection «10/10», 1985, 272 p.

L'Entourloupé, Paris, Éditions Jean Picollec, 1985, 270 p.

Le Matou, Montréal, Québec-Amérique, 1981, 584 p.

Le Matou, Montréal, Éditions du Club Québec-Loisirs, 1981, 584 p.

Le Matou, Paris, Julliard, 1982, 586 p.

Le Matou, Paris, France-Loisirs, 1982, 604 p.

Le Matou, Montréal, Les Productions le Geai Bleu inc./Éditions Québec-Amérique, Collection "Best-seller", 1985, 592 p.

Le Matou, Paris, Éditions Presses Pocket, 1986, 584 p.

Den satans katten (Traduction norvégienne du Matou, par Axel Amlie), Oslo, Aschehoug, 1984. $466 \mathrm{p}$.

Hankatten (Traduction danoise du Matou, par Karen Mathiasen), Copenhague, Chr. Erichsens Forlag, 1985,376 p.

Katti (Traduction finnoise du Matou, par Jukka Mannerkorpi), Helsinki, Kustannusosakeyhtio Otava, $1985,542 \mathrm{p}$.

Ogato (Traduction portugaise du Matou, par Octavio Mendes Cajado), Sao Paulo, Difel, 1985. 544 p.

The Alley Cat [Traduction anglaise du Matou, par Sheila Fishmann], Toronto, McClelland and Stewart, 1986, $450 \mathrm{p}$.

\section{Contes et nouvelles}

Cybèle (Avec une postface de Jacques Godbout et des sérigraphies de Cosgrove, L'Archevêque, Le Sauteur, Masson et Tanobe), Montréal, Art Global, 1982, 48 p.

"Idylle 1913", Sept-Jours, no 28, 25 mars 1967, p. 29.

(Jiviri Marxovitz, pseudonyme), "Je vous étranglerai avec mon caleçon", Bulletin $S G E R Q$ (Syndicat général des employés de Radio-Québec [CSN]), Montréal, 29 mai 1978 ou 25 septembre 1978, $18 \mathrm{p}$.

«Le Premier Noël du Père Noël», Sept-Jours, no 15, 24 décembre 1966, p. 31-33.

«L'Etrange sommeil de Jérémie Jérôme», Sept-Jours, no 17, 7 janvier 1967, p. 33.

"Le Vieux capitaine", Télé-Clé (Radio-Québec), vol. 3, no 2, mars-avril-mai 1975, p. 26-27.

"Sueurs", dans Fuites et Poursuites, Montréal, Quinze, 1982, 208 p.

«Sueurs», dans Fuites et Poursuites, Montréal, Quinze, Collection « 10/10», 1985, 224 p.

«Un médecin débrouillard», Sept-Jours, no 18, 14 janvier 1967, p. 35.

“Un monument à Claude-Henri Grignon", Sept-Jours, no 27, 18 mars 1967, p. 28-29.

et al. - La Vache et d'autres animaux (pour enfants). Montréal, les Editions la Courte Échelle, 1982,24 p.

Films

Burlex (un film d'Yves Beauchemin), 24 minutes, noir et blanc, $16 \mathrm{~mm}$. Une production de L.F.B. Via Le Monde Canada Inc., Montréal, 1971.

Le Matou (un film de Jean Beaudin d'après le roman de Yves Beauchemin), 133 minutes, en couleurs. Une production de Justine Héroux, Montréal, 1985. Distribution: Vivafilm. 


\section{Articles divers}

"Author says his book not intended to be anti-Semitic" (lettre), The Gazette, December 11 th 1981, p. 6.

"Courrier du lecteur", Longueur d'onde (Magazine de Radio-Québec), vol. 1, no 5, décembre $80 /$ janvier 81 , p. 18 .

"Défense du fédéralisme», Dimensions, vol. 6, no 4, mai 1969, p. 30-31.

"En quête de la modernité», Possibles, vol. 8, no 3, printemps 1984, p. 117-119.

"Être écrivain: un métier comme les autres?", le Devoir, 18 novembre 1978, p. 3.

"Journal intime (extraits)", Possibles, vol. 9, no 3, printemps 1985, p. 137-148.

"L'Affichage français" (manuscrit), Mémoire à la Commission permanente des communautés culturelles et de l'immigration. Montréal, 7 octobre 1983, 12 p. Présenté oralement le 4 novembre 1983. Voir le Journal des Débats, Commissions parlementaires, $4^{\mathrm{e}}$ session, $32 \mathrm{e}$ Législature, Audition de personnes et d'organismes sur la Charte de la langue française (8), le vendredi 4 novembre 1983 , no 162 , p. B-9008 à B-9011.

"La Littérature québécoise par ses écrivains - en attendant le feu d'artifice», Liberté, vol. 19, no 3 (no 111), mai-juin 1977, p. 18-22.

"La Ville, la musique et le reste", le Devoir, 20 novembre 1982, p. 6.

"Le Crâne de monsieur Duchêne" (extrait du Matou), le Devoir, 24 novembre 1979, cahier 3, p. 2.

"Le Journal intime d'Yves Beauchemin», Service des transcriptions et dérivés de la radio, Maison de Radio-Canada, cahier no 9, $42 \mathrm{p}$.

"Le Pays-bonbon", Québec-Amérique, vol. 4, no 8, 1982, p. 14-15.

"Les Média - l'information littéraire: une faillite!", l'Actualité, vol. 3, no 2, février 1978, p. 6. "L'Exemple du Matou" (lettre), le Devoir, 27 février 1982, p. 17.

"L'Humour et la littérature" (texte inédit). Série: Conférence sur un banc de parc. Donné au Salon du livre. 2 décembre 1979, $7 \mathrm{p}$.

"Naissance d'un pays» (texte inédit). Conférence prononcée le ler avril 1978 à New York à l'Alliance française devant les membres de la Société des professeurs de français en Amérique (S.P.F.A.), 16 p.

"Question de besoins" (lettre), le Devoir, 18 juin 1985, p. 8.

"Stop finding in my work ideas that I find nauseating", The Gazette, September 11 th 1985 , p. B-3.

«Un enfant de village» (manuscrit d'un texte radiophonique), juillet 1978, 27 p. Diffusé le 17 janvier 1979 dans la série «Un écrivain et son pays» (CBF-FM). Réalisation: Louis-Philippe Hébert.

«Un petit pas en avant... deux pas derrière» (lettre), le Journal de Montréal, 10 septembre 1984, p. 16.

Journal

Du sommet d'un arbre, Montréal, Québec-Amérique, 1986, 140 p.

\section{ARTICLES SUR YVES BEAUCHEMIN ET SES OEUVRES}

\section{Cybèle}

BLANCHARD, Louise, "Exposition de livres d'art à la Bibliothèque nationale», le Journal de Montréal, 6 décembre 1982, p. 39.

CORRIVAULT, Martine R., "De beaux livres pour les rudes soirs d'hiver", le Soleil, 4 décembre 1982, p. D-9.

DAIGNEAULT, Gilles, "Art global", le Devoir, 4 décembre 1982, p. 32.

\section{Fuites et poursuites (sueurs)}

COSSETTE, Gilles, "Signes et Pistes II - Fuites et Poursuites", Lettres québécoises, no 28 , hiver $1982-1983$, p. 32-33. 
LAPIERRE, René, «À qui le crime profite", le Devoir, 23 octobre 1982, p. 21 et p. 36.

MARTEL, Réginald, "Dix écrivains, des tas de morts. Le stylo des assassins", la Presse, 4 septembre 1982 , p. C-3.

TASCHEREAU, Yves, "Dix auteurs québécois jouent aux gendarmes et aux voleurs", Livre d'ici, vol. 7, no 51, 22 septembre 1982.

\section{L'Enfirouapé}

DUHAIME, Colette, "Yves Beauchemin. Un rêve à réaliser", le Droit, 11 mars 1978, p. 18.

ÉTHIER-BLAIS, Jean, «la Quadrature du cercle de la défaite», le Devoir, 15 juin 1974, p. 17.

FERRON, Jacques, "Un enfirouâpé, pas d'enfirouâpète", Québec-Presse, 2 septembre 1974, p. 56.

GODBOUT, Jacques, «les Livres - quinze ans plus tard», le Maclean, vol. 14, no 8, août 1974, p. 7-8.

LAROCHE, Maximilien, "Yves Beauchemin. L'Enfirouapé", Livres et Auteurs québécois 1974, Québec, Les Presses de l'Université Laval, 1975, p. 46-48. Voir aussi p. 388.

MAISONNEUVE, Lise, "Trois romans d'octobre", Prétexte, "Octobre 70/Dix ans", vol. 2, no 1 , octobre 1980 , p. 16-23. Voir aussi p. 75.

MARTEL, Réginald, "la Terreur qui répand le sourire», la Presse, 27 avril 1974, p. E-3.

RICARD, François, "L'Enfirouapé: une fête tragique", le Jour, II et 12 mai 1974, p. V-3.

SUGDEN, Leonard W., "Quebec's Revolutionary Novels", Canadian Literature, No. 82, Autumn 1979, p. 133-141.

THERIAULT, Jacques, "L'Enfirouapé de Beauchemin", le Devoir, 13 avril 1974, p. 12.

ANONYME, “Beauchemin (Yves). L'Enfirouapé», le Livre canadien, vol. 5, juin 1974, article no 192.

ANONYME, «le Rayonnement des Québécois - arts et lettres", le Québec en bref, vol. 10, no 2, février 1976, p. 12.

ANONYME, «Prix littéraire France-Québec à Yves Beauchemin et Louise Déchêne”, la Presse, 28 mai 1975 , p. B-15.

ANONYME, "Prix littéraires. France-Québec", Vient de paraitre, vol. 11, no 3, septembre 1975, p. 17.

\section{Le Matou}

Cette section se subdivise en deux parties: les articles consacrés principalement au roman, et ceux axés surtout sur le film. Il va de soi que certains d'entre eux abordent à la fois l'un et l'autre sujet.

\section{- Le roman}

ANTOINE, Alain, «Louis Caron et Yves Beauchemin. Natifs du Québec, ils parlent des longs fleuves et de la vie pleine de rires n, la Dernière Heure (Bruxelles), 77 e année, no 69, 10 mars 1982 , p. 16.

AUDET, Noël, "Le Matou. Une fête du récit», le Devoir, 4 juillet 1981, p. 15.

BARBRY, François-Régis, "Le Matou, par Yves Beauchemin », la Vie (France), no 1916, 20 au 26 mai 1982, p. 11.

BEAULIEU, Michel, "Yves Beauchemin. Un iściuiviste à l'affût de la vie quotidienne... ", Livre d'ici, vol. 6, no 42, 22 juillet 1981. Voir aussi le Nouveau Clairon (Saint-Hyacinthe), 7 octobre 1981, p. 43, et le Joliette, 7 octobre 1981, p. D-3 et p. D-13.

BÉLIL, Michel, «Barcelo et Beauchemin: romanciers de la littérature générale”, Imagine (Revue de science-fiction québécoise), no 12 (vol. 3, no 3), printemps 1982, p. 55-56.

BOIVIN, Jean-Roch, "les Livres du mois», Montréal ce mois-ci, vol. 8, no 5, mai 1982, p. 12.

BORDEAU, Julia, "Yves Beauchemin: une vraie fête", Figaro Magazine (Paris), 5 juin 1982, p. 70.

BOUCHARD, Martial, "Pour mieux vivre l'automne», Peuple-Tribune, $7^{\mathrm{e}}$ année, no 40, 7 octobre 1981, p. A-25. 
BOURASSA, Louise, "l'Auteur du Matou, un gars de chez nous», Joliette Journal, 9 octobre 1985, p. 80.

BOURDIER, Jean, "Le Matou d'Yves Beauchemin. La joie d'écrire", Minute (Paris), no 1044, 12 au 18 avril 1982, p. 24.

CADOTTE, François, "Le Malou: une bonne recette trop épicée», Liaison St-Louis, $9^{\circledR}$ année, no 29, 16 octobre 1985, p. 18.

CAGNON, Maurice, "Yves Beauchemin: Le Matou», French Review, Vol. 55, No.6, May 1982, p. 920-921.

CELLARD, Jacques, «Sous le regard de quatre romanciers. Québec 1948-1982: le changement ", le Monde (Paris), 28 et 29 mars 1982, p. 1 et p. 9.

CORRIVEAU, Hugues, «Mistigri ou le roman parfait. Le Matou de Yves Beauchemin», Spirale, no 21 , septembre 1981, p. 10.

CÔTÉ, Claire, «Commentaires le Matou», Nuit blanche, Printemps-été 1982, p. 5-6.

CÔTÉ-LEVESQUE, Corinne, "Ces chers petits poches», le Journal de Montréal, 10 septembre 1985, p. 50.

DE COMBEROUSSE, Françoise, "le Québec annexe Paris», France-Soir (Paris), 30 avril 1982, p. 9.

DEMERS, Dominique, “Le virus du best-seller», l'Actualité, Décembre 1986, p. 142-148.

DENIS, Fernand, "Pourquoi pas un roman québécois? Le Matou, c'est l'fun", la Cité (Bruxelles), 20 et 21 mars 1982, p. 9.

DESCHÃTELETS, Louise, "À lire cet été: Le Matou... du plateau", le Journal de Montréal, 17 juillet 1984, p. 42.

DESJARDINS, Normand, "Notre choix. Le Matou de Yves Beauchemin, le livre du mois choisi par le comité de rédaction", Nos Livres, vol. 12, août-septembre 1981. Voir aussi "Le Matou", article no 323 de la même revue.

DESROSIERS, Yvon, "Mythes et symboles fondamentaux dans la littérature québécoise", Canadian Issues/Thèmes canadiens («Religion/Culture. Comparative Canadian Studies. Etudes canadiennes comparées»), vol. 7, 1985, p. 232-245.

DE VESTER, Suzanne, "Double feu d'artifice québécois", Libre Belgique (Bruxelles), 99e année, no 76, 17 mars 1982, p. 17.

DORION, Gilles, "Romans. Le Matou, Yves Beauchemin", Québec français, no 43, octobre 1981, p. 10.

DUFRESNE, Jacques, "le Matou et le mouton", la Presse, 30 août 1985, p. A-6.

DUFRESNE, Jacques, "Pour un retournement populaire et un redressement de l'État», dans Crise et Leadership: les organisations en mutation, Boréal Express, Montréal, 1983, p. $37-55$ (surtout p. 38-40).

DUPUIS, Gilbert, «Le Matou (ou le plaisir de lire)», le Rimoushois, vol. 14, no 44, 16 septembre 1981, p. AA-2.

ESCOMEL, Gloria, “Une đuvre controversée; le Matou», Jonathan, no 10, février 1983, p. 24-25.

FILION, Pierre, "Les années 80", Petite histoire de l'édition québécoise, 22 mars 1984, p. 1-4.

FRASER, Graham, "Can anti-Semitism in Quebec be rooted out?", The Gazette, November 3rd 1981, p. 7.

FRASER, Graham, "Quebec's in a Tomcat mood", The Globe and Mail, September 21 st 1985, p. 8.

GAGNON, Lysiane, "Dis-moi ce que tu lis... ", la Presse, 14 août 1982, p. A-7.

GAGNON, Maurice, "Le Matou d'Yves Beauchemin: une lecture idéologique», l'Esprit créateur, Fall 1983, p. 95-104.

GARBACHEVSKI, Franz, "Des fleurs à Yves Beauchemin» (lettre d'un lecteur), la Presse, 18 actobre 1982, p. A-7.

GARCIN, Jérôme, “les Québécois arrivent... Le Montréal d'Yves Beauchemin vaut mieux que le Paris de Roger Fournier", les Nouvelles littéraires (Paris), $60^{\mathrm{e}}$ année, no 2827, 11-18 mars 1982, p. 40-41. 
GASCHT, André, "Yves Beauchemin: Le Matou. Certes de quoi fouetter un chat", le Soir (Bruxelles), 96 ${ }^{\circledR}$ année, no 62, 16 mars 1982 , p. 18.

GAUTHIER, Guy, "Le Matou par Yves Beauchemin", Pourquoi? (La revue de l'éducation permanente), no 169, novembre 1981, p. 91-92.

GAUTHIER, Yves, "Le Matou», le Troisième Âge, novembre 1981, p. 18.

GENUIST, Monique, "Cinéaste, moraliste. Yves Beauchemin: Le Matou. Agnès Guitard: Les Corps communicants", Canadian Literature, No. 92, Spring 1982, p. 111-113.

GIRARD, Jean-Pierre, "Auteur du livre Le Matou, Yves Beauchemin, craint l'accueil pour son prochain roman ", le Point (Dolbeau), 7 e année, no 16, 24 février 1982, p. 9.

GIROUARD, Pierre, "Yves Beauchemin: un écrivain qui ne craint pas de montrer sa griffe", les 2 Rives, 20 décembre 1983, p. 29 et p. 31.

GRAINVILLE, Patrick, "Le Matou d'Yves Beauchemin", VSD (Paris), no 239, 1 au 7 avril 1982, p. 58.

GRAMONT, Monique de, "Yves Beauchemin. Un Matou sur la conscience», Châtelaine, Janvier 1987 , p. $18-22$.

GRENIER, Serge, "Vicieux Matou, va!», Plus, 12 février 1983, p. 2.

GUTH, Paul, "Le Matou par Yves Beauchemin", la Voix du Nord (France), ll mai 1982, p. 20.

HAFSIA, Jélila, "Le Matou d'Yves Beauchemin. Du charme et de la fantaisie", la Presse (Tunis), 13 juin 1983.

JACQUEMART, Fabrice, «Livres du Québec: l'histoire de Caron et les histoires de Beauchemin", Journal et Indépendance Aujourd'hui (Gosselies, Belgique), no 89, $145^{\mathrm{e}}$ année, 30 mars 1982, p. 7.

JOHNSON, William, "An old-fashioned novel packs a novel punch", The Globe and Mail. June 19th 1981, p. 5.

"LACOMBE, Louise, «De la rencontre avec Yves Beauchemin ", l'Écho de Louiseville-Berthier, 10 novembre 1982, p. 23.

LACOMBE, Louise, "Le Matou: un livre pas comme les autres. Son auteur: un écrivain accessible, un homme sans prétention", l'Écho de Louiseville-Berthier, 13 octobre 1982 , p. 17.

LAFRANCE, Hélène, "Lis tes ratures. Le Matou, une fête de l'imagination", le Collectif, vol. 5 , no 7,16 novembre 1981, p. 8 .

LAMON, Georges, "Une autre palme pour le Matou. Le prix du public va à Yves Beauchemin», la Presse, 26 novembre 1985, p. G-1.

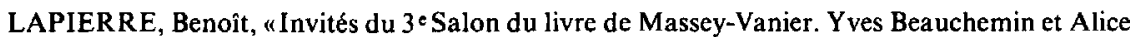
Parizeau: deux auteurs qui 'marchent' bien", la Voix de l'est, 16 novembre 1985, p. 23.

LAVIGNE, Nicole, "le Secret du Matou: un univers signé Beauchemin", l'Express Rive-Sud, no 7 , automne 1983, p. 18-20.

LEROUX, Pierre, "Au Salon du livre", le Journal de Montréal, 26 novembre 1985, p. 45.

LEROUX, Pierre, "René Lévesque au lancement du Matou en livre de poche", le Journal de Montréal, 21 août 1985, p. 62 .

P. L., "Succès non démenti pour le $13^{\mathrm{e}}$ rendez-vous littéraire. Le Prix du livre de l'été à un Canadien ", l'Avenir-L'indépendant (France), 24 au 30 juillet 1982, p. 10.

MALO, Reine, "l'Univers du Matou. Reine découvre et apprivoise Yves Beauchemin", Coup de pouce, vol. 2, no 11, 27 décembre 1985, p. 48-49.

MANGUEL, Alberto, "From the golden age, a novel with hope. Le Matou by Yves Beauchemin", The Globe and Mail, January 15th 1983, p. Entertainment 13.

MARCOTTE, Gilles, "Une sacrée bonne année littéraire», l'Actualité, vol. 6, no 8, août 1981, p. 58.

MARTEL, Réginald, “Chez Québec-Amérique», la Presse, 16 septembre 1985, p. B-5.

MARTEL, Réginald, "Le Matou de Beauchemin. La joyeuse chronique d'une jeunesse qui rêve... et calcule ", la Presse, 25 avril 1981, p. C-3.

MARTEL, Réginald, “Littérature. Chat échauđé...», la Presse, 2 mai 1983, p. A-9. 
MARTEL, Réginald, "Littérature. De tout en bref», la Presse, 31 janvier 1983, p. C-3.

MARTEL, Réginald, "Littérature. De tout en bref ", la Presse, 7 février 1983, p. B-5.

MARTEL, Réginald, "Littérature. De tout en bref", la Presse, 21 février 1983, p. B-4.

MARTEL, Réginald, "Une œuvre déjà et vive la musique. Yves Beauchemin, écrivain", la Presse, 8 janvier 1983, p. C-1 et p. C-3.

MARTIN, Jean-Guy, «les Finalistes du Grand Prix littéraire du Journal de Montréal et du Prix des jeunes écrivains ", le Journal de Montréal, 21 novembre 1981, p. 54.

MARTIN, Jean-Guy, "Yves Beauchemin. Lauréat du Prix des jeunes auteurs du journal», le Journal de Montréal, 5 décembre 1981, p. 40.

MARTIN, Jean-Guy, "Yves Beauchemin: 'Pour moi, écrire c'est avant tout raconter une histoire'», le Journal de Montréal, 25 avril 1981, p. 46.

MATHIEU, André, "Ā propos du Matou», le Soleil, 31 mai 1986.

MÉLANÇON, Robert, "Littérature québécoise. André Major: la Folle d"Elvis; Yves Beauchemin: Le Matou", Liberté, no 142, vol. 24, no 4, juillet-août 1982, p. 80-83.

MOURET, José, "Des livres et des romans. Un surprenant «Matou" québécois», ParisNormandie, 9 avril 1982, p. 12.

NAUDIN, Odile, "Le Matou», la Croix (Paris), 102' année, no 30163, 2/3 mai 1982, p. 19.

NERON, Carol, «Fourre-tout... Lancement de livre», le Quotidien (Chicoutimi), 10 février 1982 , p. 12.

NÉRON, Carol, "Fourre-tout... Le Matou à Alma", le Quotidien (Chicoutimi), 23 février 1982 , p. 12.

NOURISSIER, François, "Littérature francophone. Vive le Québec livres! ", le Point (Paris), no 501, 26 avril 1982, p. 129-131.

NURIDSANY, Michel, "Conte canadien pour grandes personnes", le Figaro (Paris), 8 avril 1982, p. 24.

PAINCHAUD, Clotilde T. L., «Événements. Yves Beauchemin au Mardi littéraire du 29 mars 1983 ", Grimoire, avril 1983, p. 8.

PARENT, Paul-André, "Un cheminement imprévu», l'Éclaireur-Progrès (Saint-Georges de Beauce), vol. 75, no 52. 14 septembre 1983, p. A-23.

PARISIEN, Thérèse, "Yves Beauchemin. Les sombres prédictions d'un romancier", la Semaine, vol. 2, no 6, 6 octobre 1984, p. 16-18.

PARIZEAU, Alice, "Un romancier qui exprime son amour avec ses personnages", la Presse, 17 mars 1986, p. B-4.

PAULIN, Marguerite, "les Femmes du Matou» (lettre d'une lectrice), l'Actualité, vol. 8, no 4, avril 1983, p. 4.

PELLETIER, Denise, "Pour Yves Beauchemin - la France après le Saguenay-Lac-St-Jean ", Progrès-Dimunche (Chicoutimi), 21 février 1982, p. 53.

PELOQUIN, Ginette, "Ecrits. Yves Beauchemin. Le Matou», Focus, no 45, octobre 1981, p. 64 .

PEREL, Shloime, et SREBERNIK, Henry, «Signes des temps", le Devoir, 17 février 1982, p. 9. (Traduction de l'article, "Signs of the Times", paru dans The Jerusalem Post, January 22nd 1982, p. 12.)

PERRAULT, Yvan, «la Littérature québécoise à son meilleur: le Matou», le Matulu, vol.6, no 3 , octobre 1983, p. 2.

PERREAULT, Luc, "Yves Beauchemin: 'J'écris comme je vois la vie'», Québec-Amérique, vol. 3, no 5/6. 1981, p. 10-11.

PERREAULT, Pascale, "les Lauréats du Journal de Montréal: deux auteurs de QuébecAmérique", le Journal de Montréal, 25 novembre 1981, p. 74.

PETROWSKI, Nathalie, "Yves Beauchemin", En Route (Air Canada), vol. 13, no II, novembre 1985 , p. 58-59 et p. 91-95.

POULIN, Gabrielle, "Le Matou de Yves Beauchemin. Un roman avec des griffes", le Droit, 8 août 1981, p. 14. 
POULIN, Gabrielle, "le Père est mort; vive le parrain! Le Matou de Yves Beauchemin », Lettres québécoises, no 23, automne 1981, p. 17-19.

POURCEL, Gérard, "Le Matou de Yves Beauchemin chez Québec-Amérique », le Lac St-Jean, 10 février 1982, p. 24.

POURCEL, Gérard, «les Livres de l'été. Prix de la Ville de Montréal. Le Matou. Yves Beauchemin ", le Lac St-Jean, 22 juin 1982, p. 20.

POURCEL, Gérard, "Yves Beauchemin à Alma. Un marathon électoral", le Lac St-Jean, 24 février 1982, p. 25.

POZIER, Bernard, “Un livre en cadeau?», le Nouvelliste, 19 décembre 1981, p. 20.

QUEFFÉLEC, Yann, "Lettres québécoises. Mystère et boule de gomme. Comment un drame peut rester émouvant tout en étant farfelu», le Nouvel Observateur (Paris), 5 ou 11 juin 1982, p. 56.

ROBERT, Véronique, "Le Matou c'est lui", l'Actualité, vol. 8, no 2, février 1983, p. 32-37.

ROBILlARD, Benoit, "Yves Beauchemin, écrivain", Commerce Rive-Sud, vol. 7, no 4, septembre-octobre 1983, p. 5.

ROSSET, Pierrette, «Attention, plaisir fou!", Elle (Paris), no 1894, 26 avril 1982, p. 59.

ROY, Pierrette, «Yves Beauchemin: un écrivain accessible», la Tribune (Sherbrooke), 4 juillet 1981, p. C-2.

ROYER, Jean, «la Revue des revues», le Devoir, 5 février 1983, p. 21.

ROYER, Jean, "la Vie littéraire», le Devoir, 20 mars 1982, p. 19.

ROYER, Jean, «la Vie littéraire", le Devoir, 22 janvier 1983, p. 14.

ROYER, Jean, «la Vie littéraire». le Devoir, 31 août 1985, p. 22.

ROYER, Jean, "la Vie littéraire. Quand le chat sort du sac", le Devoir, $1^{\mathrm{er}}$ mai 1982, p. 22.

ROYER, Jean, «le Nouveau souffle littéraire. Quelques labels de qualité », le Devoir, 8 janvier 1983, p. 13 et p. 24.

ROYER, Jean, «les Plaisirs de la terre-fiction», le Devoir, 4 juillet 1981, p. 13-14. Voir aussi "Yves Beauchemin", dans Ecrivains contemporains. Entretiens 3: 1980-1983, Montréal, L'Hexagone, 1985, p. 145-154.

ROYER, Jean, "Parmi les dernières parutions», le Devoir, 12 décembre 1981, p. 30.

ROY-GUERIN, Michelle, “Le Matou... ", le Nouvelliste, 6 août 1983, p. 5A.

RUDEL-TESSIER, Danièle, "De beaux livres pour Noël... ", l'Actualité, vol. 6, no 12, décembre 1981, p. 136.

SMITH, Donald, «Yves Beauchemin ou le goût de l'action, du baroque et du gigantisme: une entrevue avec Donald Smith", Québec-Amérique (Bulletin d'information), vol. 1, no 5, octobre 1985, p. 3-5.

STRATFORD, Philip, "Matou as satiating as a sumptuous meal", The Gazette, February 19 th 1983 , p. B-7.

M.T., «Un invité de marque au 26 ième Salon du livre», le Ciroyen (Asbestos), vol. 44, no 51 , 24 avril 1984, p. 2.

TREMBLAY, Régis, "Ce qui est généreux m’attire...», le Soleil, 10 décembre 1983, p. E-8.

TREMBLAY, Régis, "Le Matou a le vent dans les poils", le Soleil, 18 mai 1985, p. C-9.

TREMBLAY, Régis, "Yves Beauchemin et Agnès Guitard, deux auteurs de la couvée romanesque de Québec-Amérique ", le Soleil, 6 juin 1981, p. E-3.

TRUDEL, CLément, "Andréanne Lafond. L'art de l'interview», le Devoir, 18 juillet 1981, p. 13.

TRUDEL, CLément, «Le Matou, Le Parrain et les autres. A quoi tient, au Québec, la littérature à succès?", le Devoir, 28 avril 1984. p. 58.

TURBIDE, Diane, "Two Solitudes", Saturday Night, Vol.99, No. 12, Whole No. 3657, December 1984, p. 59-60.

TURNER, Marie Linda, "En tête à tête avec Yves Beauchemin", Liaison St-Louis, 24 mai 1984, p. 10.

VANASSE, André, “Roman. Turgeon, Beauchemin, Tremblay et les autres...», Voix et images, vol. 7 , no 2, hiver 1982, p. 417-419. 
VANASSE, André, "Yves Beauchemin. Le Matou», Livres et Auteurs québécois 1981, Québec, Les Presses de l'Université Laval, 1982, p. 26-28. Voir aussi p. 354.

WEINSTOCK, Daniel M., "Une entrevue avec Yves Beauchemin: Écrire à temps complet, un rêve pour un écrivain québécois», le McGill Daily, 10 novembre 1981, p. 2.

XENAKIS, Françoise, "Cosmo livres. Le Matou. Yves Beauchemin", Cosmopolitan (Paris), no 104 , juillet 1982, p. 20.

XENAKIS, Françoise, "Devoirs de vacances de Michel Pérez et Le Matou d'Yves Beauchemin», le Matin de Paris, 5 avril 1982, p. 21.

ZAND, Nicole, "le Québécois, ce n'est pas une langue, c'est une musique ", le Monde (Paris), 26 et 27 juin 1983, p. 12. Voir aussi le Devoir, 2 juillet 1983, p. 14.

ANONYME, «Au Québec», la Presse, 9 septembre 1985, p. B-7.

ANONYME, "Beauchemin lauréat du Prix littéraire de la CUM", la Presse, 29 avril 1982, p. D-2.

ANONYME, "Beauchemin reçoit le 'Prix du livre de l'été"», la Presse, 17 juillet 1982, p. A-2. Voir aussi « Pour Yves Beauchemin, le Prix du livre de l'été", le Droit, 16 juillet 1982, p. 23; "Yves Beauchemin reçoit le Prix du livre de l'étén, le Soleil, 16 juillet 1982, p. A-9, le Nouvelliste (Trois-Rivières), 16 juillet 1982, p. 22, l'Évangéline (Moncton), 16 juillet 1982, p. 13; et "Yves Beauchemin reçoit le 'Prix du livre de l'été' à Cannẹs en France», le Quotidien (Chicoutimi), 16 juillet 1982, p. 13.

ANONYME, "Best-sellers du livre", le Journal de Montréal, 25 octobre 1981, p. 64.

ANONYME, «Bookprize for Beauchemin», The Gazette, April 29th 1982, p. D-11.

ANONYME, "Grand Prix litțéraire», Progrès-Dimanche (Chicoutimi), 2 mai 1982, p. 69.

ANONYME, "Grosses légumes et légumineuses au lancement du Matou», Liaison St-Louis, 9 e année, no 23, 4 septembre 1985, p. 5 .

ANONYME, «la Critique unanime», Québec-Amérique, vol. 4, no 8, 1982, p. 5.

ANONYME, «la Critique unanime! Le Matou» (Publicité), le Devoir, 10 octobre 1981, p. 7.

ANONYME, “la Fête du 'mịni' Matou», le Journal de Montréal, 27 août 1985, p. 34.

ANONYME. "Le Matou", Culturama (Programmes de Culture Canadienne L.A.E. Inc.), vol. 123, février 1986, p. 1-2.

ANONYME, "Le Matou en format de poche», le Droit, 20 août 1985, p. 11, Voị ausși " $L e$ Matou sort en format de poche", le Soleil, 24 aout 1985, p. C-8.

ANONYME, "Le Matou en livre de poche», le Nouvelliste, 27 août 1985, p. 23.

ANONYME, "Le Matou en livre de poche,... un lancement chaleureux et enthousiaste", Québec-Amérique (Bulletin d'information), vol. 1, no 5, octobre 1985, p. 2.

ANONYME, «Le Matou, Format de poche, lancé le 26 aoụt à la Mạison de la culture du Plateau", Liaison St-Louis, 9e année, no 21,21 août 1985, p. 2.

ANONYME, "Le Matou revient d'Europe", l'Autre presse, début mai 1982, vol. 1, no 1, p. 3.

ANONYME, "Le Matou. Yves Beauchemin", Femmes, d'aujourd"hui (Paris), no 17, 27 avril au 3 mai 1982, p. 10.

ANONYME, «le Prix de la CUM à Yves Beauchemin», le Devoir, 28 avril 1982, p. 1 et p. 8.

ANONYME, «les Finalistes du Grand Prix littéraire», le Devoir, 18 novembre 1981, p. 13.

ANONYME, "les Finalistes du Grand Prix littéraire et des jeunes écrivains du Journal de Montréal,, le Journal de Montréal, 18 novembre 1981, p. 64-65.

ANONYME, «les Prix littéraires du Journal de Montréal. Le Grand Prix: Gilbert La Rocque. Les Jeunes Écrivains: Yves Beauchemin", le Journal de Montréal, 25 novembre 1981, $\mathrm{p}_{\text {. }}$ I et p. 66-67.

ANONYME, "Lévesque a rencontré Monsieur Émile", la Presse, 27 aột 1985, p. A-4.

ANONYME, "Quelques nouveautés. Yves Beauchemin. Le Matou", la Semaine du livre, vol, 1 , no 6, 10 au 19 avril 1981 , p. 13.

ANONYME, «Rights», Quill \& Quire, March 1983, p. S3.

ANONYME, "Un dactylo en or massif: Yves Beauchemin", Livre d'ici, novembre 1982, p. 3.

ANONYME, "Une griffe célèbre», le Devoir, 27 août 1985, p. 1. 
ANONYME, "Une carrière fulgurante pour Le Matou» (Traduction de l'article "Rights", Quill \& Quire, March 1983, p. 53), Québec-Amérique, vol. 5, no 9, avril-mai 1983, p. 56.

ANONYME, “Yves Beauchemin lauréat du 'Prix du livre de l'été'», le Devoir, 19 juillet 1982 , p. 3. Voir aussi «Yves Beauchemin reçoit le 'Prix du livre d'été' pour Le Matou», le Journal de Montréal, 18 juillet 1982, p. 36.

ANONYME, "Yves Beauchemin. Le Matou», Québec-Amérique, vol. 3, no 7, 1981, p. 4-5.

ANONYME, "Yves Beauchemin. Le succès du Matou», Lettres québécoises, no 29, printemps 1983 , p. 13.

ANONYME, "Yves Beauchemin reçoit le prix du Journal de Montréal", Flash (Direction des communications Radio-Québec), vol. 8, no 58, 26 novembre 1981.

\section{- Le film}

ACKERMAN, Marianne, "Le Matou jumps from book to screen», The Gazette, August 28 th 1985 , p. C-7.

ACKERMAN, Marianne, "Le Matou's rhythm is lost on film», The Gazette, August 29th 1985. p. D-10.

ALEMANY-GALWAY, Mary, “Jean Beaudin's Le Matou», Cinéma Canada, No. 126, January 1986, p. 33-34.

BAZZO, Marie-France, "Monsieur Guillaume-Emile-Lemay-Le Matou-Thivierge...n, Ciné Festival (Le quotidien officiel du 9e Festival des films du monde de Montréal), no 8, 28 aout 1985, p. 4.

BAZZO, Marie-France, "Oeil sur la programmation", Ciné Festival (Le quotidien officiel du $9^{\mathrm{e}}$ Festival des films du monde de Montréal), no 8,28 août 1985, p. 2.

BAZZO, Marie-France, "Un seul absent: le chat", Ciné Festival (Le quotidien officiel du $9^{\text {e }}$ Festival des films du monde de Montréal), no 9,29 août 1985, p. 11.

BERNATCHEZ, Raymond, “Denis Héroux en assurera la production. Deux versions du Malou tournées prochainement", la Presse, 22 avril 1984, p. 83.

BLACK, Barbara, "Le Matou, Mario are good stories and fodder for film director". The Gazette, September 7th 1985, p. C-3.

BONNEVILLE, Léo, "Entretien avec Jean Beaudin", Séquences (Revue de cinéma), no 118. octobre 1984, p. 5-9.

BONNEVILLE, Léo, «Le Matou», Séquences (Revue de cinéma), no 122, octobre 1985, p. 38-39.

BOULAD. Bernard, "Le Matou en compétition. Un fauve dans l'arène", Ciné Festival (Le quotidien officiel du $9^{\text {e }}$ Festival des films du monde de Montréal), no 8, 28 août 1985, p. 5.

BOULAD, Bernard, "Oeil sur la programmation", Ciné Festival (Le quotidien officiel du 9 e Festival des films du monde de Montréal), no 9, 29 août 1985, p. 3.

BROUSSEAU, P., "Elle sera la 'Rosine' du Matou. Isabel a reçu deux offres de films», ÉchosVedettes, 2 au 8 septembre 1984, p. 23.

CADOTTE, François, «le Festival des films du monde: pour rassasier les cinéphiles », Liaison St-Louis, $9^{\mathrm{e}}$ année, no 21,21 août 1985 , p. 8.

CADOTTE, François, et CHOQUETTE, Gilles, «le Festival des films du monde: un événement qui fait courir", Liaison St-Louis, $9^{\mathrm{c}}$ année, no 23, 4 septembre 1985, p. 13.

CHOQUETTE, Gilles, «Dans Le Matou, selon le cinéaste Jean Beaudin, l'univers reconstitué du Plateau Mont-Royal", Liaison St-Louis, $9^{e}$ année, no 22, 28 août 1985, p. 1.

CÔTE-LEVESQUE, Corinne, "Matou-1", le Journal de Montréal, 10 septembre 1985, p. 50.

DUSSAULT, Serge, «Le Matou. $\$ 80000$ en sept jours!", la Presse, 6 septembre 1985, p. C-1.

FOGLIA, Pierre, "Cinéma", la Presse, 31 août 1985, p. A-5.

GAUDREAULT, Léonce, "Le Matou séduit le public et Les Amants de Maria la critique», le Soleil, 3 septembre 1985 , p. C-3.

GAUDREAULT, Léonce, «Pour la première du Matou l'atmosphère était à la fête au Grand Théâtre", le Soleil, 30 août 1985, p. A-3. 
GIGUÈRE, André, “Cinéma ", Montréal ce mois-ci, vol. 11, no 9, septembre 1985, p. 14.

JEAN, Marcel, "'Le Matou n'est pas un film de festival' - Jean Beaudin", le Devoir, 17 août 1985, p. 23.

JEAN, Marcel, “Le Matou. Pas de quoi fouetter un chat! », le Devoir, 7 septembre 1985, p. 31.

LAURENDEAU, Francine, "Le Grand Prix de Montréal va à un film déjà présenté à Cannes", le Devoir, 3 septembre 1985, p. I et p. 12.

LAURENDEAU, Francine, "Le Matou, truculence et magie en mineur», le Devoir, 29 août 1985, p. 1 et p. 10.

LEGENDRE, Thérèse, «Après L'Enfirouapé et Le Matou Yves Beauchemin prépare un autre roman et une mini-série", le Courrier du Sud, 17 septembre 1985, p. B-3.

LEGENDRE, Thérèse, "Le Matou d'Yves Beauchemin aura autant de succès en salle qu'en librairie ", le Courrier du Sud, 10 septembre 1985, p. B-1.

LEMIEUX, Louis-Guy, «Jean Beaudin: Le Matou, c'est l'histoire de l'indépendance», le Soleil, 15 septembre 1985 , p. B-1.

LEMIEUX, Louis-Guy, "l'Adaptation d'un roman peut-elle servir à la fois le cinéma et la télévision?", le Soleil, 15 septembre 1985, p. B-1.

LEMIEUX, Louis-Guy, «le Film Le Matou rend mal ce remarquable roman», le Soleil, 29 aout 1985, p. B-8.

MAROIS, Michel, «les Rendez-vous du cinéma québécois. Blind Date, suite et... ". Continuum (Journal des étudiants et des étudiantes de l'Université de Montréal), vol. 9, no 20, semaine du 10 février 1986, p. 14-15.

NUOVO, Franco, "Julien Guiomar, le cuisinier du Matou», le Journal de Montréal, 27 août 1985, p. 35.

NUOVO, Franco, "Le Matou - on tourne en septembre», le Journal de Montréal, 25 juillet 1984, p. 46.

NUOVO, Franco, "le Tout Montréal au lancement du Matou», le Journal de Montréal, 29 août 1985 , p. 56-57.

PERREAULT, Luc, «Best-sellers à l'écran. Voyez le film et lisez le livre!», la Presse, 29 mai 1982, p. C-11.

PERREAULT, Luc, “Jean Beaudin: 'J'ai tripé sur le Matou'», la Presse, 24 août 1985, p. D-1 et p. D-2.

PERREAULT, Luc, "la Qualité mais non le génie», la Presse, 3 septembre 1985, p. E-1.

PERREAULT, Luc, "Le Matou en tournage: comme une immense fresque", la Presse, 15 décembre 1984, p. E-1 et p. E-16.

PERREAULT, Luc, «Le Matou primé à Montréal et à Québec », la Presse, 3 septembre 1985, p. E-1.

PERREAUlT, Luc. «Le Matou: un accueil chaleureux", la Presse, 29 août 1985, p. Á-1 et p. A-2.

PER REAULT, Luc, «Montant record pour des droits d'auteur. Le Matou, porté à l'écran ", la Presse, 10 septembre 1982, p. A-13.

PERREAULT, Luc, «Une adaptation réussie», la Presse, 29 août 1985, p. B-2.

PETROWSKI, Minou, «le Tournage du Matou: quand un roman devient un film ", Clin d'ceil, no 53,25 janvier 1985 , p. 29-31.

PINARD, Guy, "Sur la scène de l'actualité: Yves Beauchemin», la Presse, 8 septembre 1985, p. 9.

POULIOT, Suzanne, "Le Matou», le Sablier, vol. 9, no 5, janvier 1986, p. 8.

RICHARD, Jules, «Jean Beaudin, cinéaste», le Devoir, 4 août 1984, p. 17 et p. 22.

ROBERGE, Pierre, "Avec au moins $\$ 300000$ en promotion le lancement du film Le Matou constitue un record québécois», la Presse, 13 août 1985, p. B-4.

ROBERGE, Pierre, «Le Matou: d'une tablette au grand écran...»le Droit, 13 août 1985, p. 16. Voir aussi "Une campagne de promotion de $\$ 300000$ pour le film Le Matou", le Soleil, 14 août 1985, p. C-3. 
ROBERGE, Pierre, "Yves Beauchemin a craint d'être déçu par son Matou au cinéma", le Journal de Montréal, 25 octobre 1984, p. 76. Voir aussi «Le Matou porté à l'écran. Yves Beauchemin garantit que ce sera un beau film", la Presse, 29 octobre 1984, p. B-11.

ROBERT, Claude, "Le Matou et Les Amants de Maria sont couronnés», le Journal de Québec, 3 septembre 1985, p. 36.

ROBERT, Claude, "l'Équipe du Matou fort bien accueillie", le Journal de Québec, 30 août 1985, p. 1-2.

ROY, Pierrette, "Le Matou: une expérience unique pour Serge Dupire", la Tribune (Sherbrooke), 17 novembre 1984, p. A-12.

ROY, Pierrette, "le Matou: 20 mois de tournage et 6,5 millions $\$$ de budget ", la Tribune (Sherbrooke), 17 novembre 1984, p. A-11.

ROY, Pierrette, «Un rucher bourdonnant d'activités", la Tribune (Sherbrooke), 17 novembre 1984 , p. A-10.

SCHNURMACHER, Thomas, "Duo make their debut at festive festival», The Gazerte, August 27 th 1985 , p. D-9.

SIBEROK, Martin, "Eye on the Program", Ciné Festival (Le quotidien officiel du $9^{\mathrm{c}}$ Festival des films du monde de Montréal), no 8, 28 août 1985, p. 3.

SOULIÉ, Jean-Paul, "Le Matou. La ruelle des Fortifications s'anime...". la Presse, 25 septembre 1984, p. B-1.

SOULIE, Jean-Paul, «Le Matou va faire mieux en France que Les Plouffe, Picquot vous le garantit...", la Presse, 29 août 1985, p. B-2.

SOULIE, Jean-Paul, "Tout le monde était là. Étrange dépaysement pour Le Matou», la Presse. 22 août 1985 , p. A-15.

SYLVAIN, Jean-Paul, "Déjà quatre films pour le chat... du Matou», le Journal de Montréal, 7 septembre 1985, p. 42.

SYLVAIN, Jean-Paul, “'M. Émile’ dans un film de \$2 millions avec Louise Marleau ", le Journal de Montréal, 28 août 1985, p. 55.

SYLVAIN, Jean-Paul, “Tout le gratin veut assister à la première du film Le Matou», le Journal de Montréal, 28 août 1985, p. 59.

TASCHEREAU, Yves, "Silence, on tape! Le cinéma devient-il une 'mine d'or' pour les écrivains et les éditeurs?", Livre d'ici, vol. 8, no I, novembre 1982, p. 1 et p. 15.

ANONYME, "Beauchemin visionne la 'copie 0' du Matou", le Droit, 26 août 1985, p. 12.

ANONYME, "Big launch for Le Matou”, Cinéma Canada, No. 122, September 1985, p. 61.

ANONYME, "Ce n'est que du cinéma ", la Presse, 10 novembre 1984, p. A-3.

ANONYME, "Cinéma. Denis Héroux produira Le Matou de Beauchemin", Échos-Vedettes, 15 au 21 août 1982, p. 28.

ANONYME, "De la neige pour le cinéma", le Devoir, 10 novembre 1984, p. 3.

ANONYME, "Deux prix au Matou», le Journal de Montréal, 2 septembre 1985, p. 22.

ANONYME, "Festival des films du monde: une fête d'adieu émouvante ", le Journal de Québec, 3 septembre 1985, p. 38-39.

ANONYME, "Le Matou au FFM", le Devoir, 17 juillet 1985, p. 7.

ANONYME, "Le Matou recreates beanery for publicity stunt», Cinema Canada, October 1985, p. 69.

ANONYME, «Le Matou represents Canada in filmfest", The Gazette, July 16 th 1985, p. C-8.

ANONYME, «Un gros Matou», TV Hebdo, vol. 25, no 3, 14 au 20 janvier 1984, p. 11.

\section{Divers}

ABEL, Marie-Christine, «la Passion insolite de... Un violon (d'Ingres) tchèque», le Devoir 11 janvier 1986, p. 26.

BENOIT, Jacques, "Ce qu'ils ont dit d'Yves Thériault", la Presse, 22 octobre 1983, p. A-11.

BLOUIN, Jean, «les Valeurs des jeunes", l'Actualité, vol. 9, no 5, mai 1984, p. 39-46.

BOURASSA, Louise, "Yves Beauchemin regrette le 'vieux Joliette'", Joliette Journal, 9 octobre 1985 , p. 80 . 
BOUTOT, Bruno, "Destination Montréal: guide pratique... et rusé", Autrement ("Québec. Histoires de chums et de grands espaces"), no 60, mai 1984, p. 253.

BROSSEAU, Daniel, «Hommage à René Lévesque... Le PQ lui dit au revoir», le Journal de Montréal, 28 septembre 1985, p. 1, p. 10 et p. 12.

DAVID, Michel, "la Loi 101 est essentielle, dit Beauchemin ", le Soleil, 5 novembre 1983, p. A-7.

DE COMBEROUSSE, Françoise, "Suivez le guide: c'est François Nourissier», France-Soir (Paris), 17 avril 1982, p. 87.

DE LAPLANTE, Michèle, “Un écrivain, c'est d'abord un artisan - Yves Beauchemin", le Joliette, 18 mai 1983 , p. B-9.

DESROSIERS, Sylvie, "Quand la marmite bout... ", Livre d'ici, vol. 11, no 2, octobre 1985, p. 6.

DUFRESNE, Jacques, «les Enfants et nous», la Presse, 14 juin 1985, p. A-6.

DUSSAULT, Jean-Claude, "Montréal s'affirme comme ville par excellence des festivals. Littérature", la Presse (Section "Excellence 85»), 19 octobre 1985, p. 7, p. 9 et p. 10.

ÉTHIER-BLAIS, Jean, "les Français et le roman québécois», le Devoir, 16 octobre 1982, p. 22.

GARON, Claude, "Esquisses. Rencontrer Yves Beauchemin", le Lac St-Jean, 17 février 1982, p. 22.

GARON, Claude, “Esquisses. Yves Beauchemin», le Lac St-Jean, 24 février 1982, p. 24.

GARON, Claude, "Esquisses. Yves Beauchemin nous visite», le Lac St-Jean, 10 février 1982, p. 23.

GIRARD, Jean-Pierre, «Écrivain, c'est pas payant», le Point (Dolbeau), 7 e année, no 16, 24 février 1982, p. 9.

GIROUX, Robert, "Notion et/ou fonctions de la littérature (nationale québécoise) au $\mathrm{XX}^{\mathrm{c}}$ siècle», Voix et images, vol. 5, no 1, automne 1979, p. 90 et p. 113.

GRIMALDI, Francine, "la Binerie Mont-Royal", la Presse, 25 août 1985, p. 65.

HEBERT, François, "le Noir et blanc, le bleu et le rouge", Etudes françaises, vol. 11, no 2, mai 1975, p. 116.

KAUFMAN, Michael T., "Celebrated Novels Fail to Link Canada's Separate Cultures", The New York Times, May 3 rd 1984, p. C'26.

LE COURS, Rudy, "Affichage en français. L'écrivain Yves Beauchemin presse Québec de ne pas toucher à la loi 101 », la Presse, 5 novembre 1983, p. A-4. Voir aussi «Selon l'auteur du Matou loi 101: une bouée de sauvetagen, le Droit, 5 novembre 1983, p. 19.

LE COURS, Rudy, "Johnson et la culture: 'un soutien concret à la liberté d'expression'", le Devoir, 18 novembre 1985 , p. 5.

LEGARE, Yves, "Yves Beauchemin", dans Dictionnaire des écrivains québécois contemporains. 1970-1982, Montréal, Québec-Amérique, 1983, p. 43.

LEROUX, Pierre, “Monique Proulx remporte le Grand Prix littéraire du Journal de Montréal", le Journal de Montréal, 15 novembre 1984, p. 76-77.

LEVESQUE, Gaëtan, "la Revue des revues», Voix et images, vol. 8, no 1, automne 1982, p. 174.

MARTEL, Réginald, "Autour du Salon du livre. Des histoires de prix», la Presse, 23 novembre 1981 , p. A-12.

MARTEL, Réginald, «Littérature. La littérature cette semaine», la Presse, 15 novembre 1982, p. B-6.

MARTEL. Réginald, «Petite épitaphe pour un grand prix», la Presse, 19 novembre 1983, p. E-3.

MARTEL, Réginald, «72 000 visiteurs au Salon du livre», la Presse, 14 novembre 1983, p. A-10.

MORIN, Marc, "le Monde du livre déplore la disparition du Grand Prix littéraire de Montréal", le Devoir, 19 juin 1982, p. 17 et 32.

PARENT, André, "'Rechercher la qualité d'abord' - Jacques Fortin", l'Éclaireur-Progrès (Saint-Georges de Beauce), 14 septembre 1983, p. A-22.

PERITZ, Ingrid, "City's neglect threatens heritage: group", The Gazette, June 26th 1985, p. A-3. 
POULIN, Gabrielle, «Des confidences d'écrivains», le Droit, 30 juin 1984, p. 22.

POULIN, Gabrielle, Roman du pays 1968-1979, Montréal, Bellarmin, 1980, p. 326.

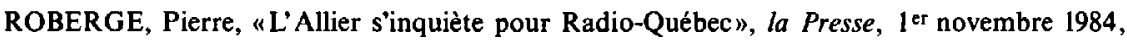
p. B-7.

ROBITAILLE, Louis-Bernard, «les Triomphes québécois à Paris. Éditer à Paris... ou ne rien vendre", la Presse, 16 août 1984, p. A-1 et p. A-2.

ROYER, Jean, "la Vie littéraire», le Devoir, 8 juin 1985, p. 22.

TEBOUL, Victor, "la Grandeur d'Israël», Jonathan, nos 12-13, avril-mai 1983, p. 3.

THÉRIAULT, Jacques, "Roman, nouvelles et contes", Livre d'ici, vol. 7, nos 12-13, 23 décembre 1981. Voir aussi l'Hebdo de Portneuf, 5 janvier 1982, p. B-8, et le Guide de Montréal-Nord, vol. 30 , no 1,6 janvier 1982 , p. 46.

WILSON-SMITH, Anthony, "Changing course in Quebec", Maclean's, Vol. 98, No. 3, January 21 st 1985, p. 14-18.

ANONYME, «Beauchemin: 'On veut enlever la cheville'», Plus (La Presse), 26 janvier 1985, p. 7.

ANONYME, "Best sellers", The Gazette, November 2nd 1985, p. D-3.

ANONYME, «le Québec à la foire", Libre Belgique (Bruxelles), 99 e année, no 69, 10 mars 1982, p. 21.

ANONYME, «le Québec pour la 6 fois», le Soir (Bruxelles), 96ªnnée, no 57, 10 mars 1982 , p. 11.

ANONYME, "le Salon du livre de Montréal - Lise Payette animatrice", le Devoir, 20 novembre 1982 , p. 15.

ANONYME, «les Ecrivains descendent dans la rue", le Devoir, 17 septembre 1983, p. 18.

ANONYME, "Meilleures ventes Paris-Province», France-Soir (Paris), 9 avril 1982, p. 11.

ANONYME, «Prix du Livre de l'Été. Le Matou» (Publicité), le Monde (Paris), 22 juillet 1982, p. 6.

ANONYME, “Reportage sur Le Matou», Québec-Amérique, vol. 1, no 6, novembre-décembre 1985, p. 2.

ANONYME, "Un imprimeur québécois à succès", le Journal de Montréal, 25 août 1983, p. 44.

ANONYME, "Yves Beauchemin. Le Matou» (Publicité), le Monde (Paris), 11 mars 1982, p. 1.

ANONYME, "Yves Beauchemin. Le Matou» (Publicité), le Monde (Paris), 2 avril 1982, p. 1.

ANONYME, "Yves Beauchemin pour Le Matou et Louis Caron pour Les Fils du soleil ont présenté leurs livres, à Liège», la Gazette (Wallonie-Liège) 2 avril 1982.

Du sommet d'un arbre

A.G., "On aime pourtant leurs confidences", le Nouvelliste, samedi 10 janvier 1987, p. 13-A.

BINET, Denis, "Description et commentaire», l'Age de son cœur, Radio-Canada, Ottawa, 11 décembre 1986.

ROYER, Jean, "L"effet d'hélicoptère'", le Devoir, 13 décembre 1986.

THERIO, Adrien, "Du sommet d'un arbre. Présentation", Lettres québécoises, Printemps 1987, no 45 , p. 8. 